\title{
Treinamento resistido para idosos: revisão de literatura
}

\author{
Resistance training for elderly: literature review
}

\author{
Socorro Fernanda Coutinho dos Santos, ${ }^{1}$ Vicente Matias da Silva Neto' \\ 'Universidade Federal do Triângulo Mineiro (UFTM), Minas Gerais, Brasil.
}

Recebido em: 27/08/2016 / Aceito em: 17/02/2017 / Publicado em: 31/03/2017

fernanda.coutinho.santos@hotmail.com

\section{RESUMO}

No Brasil, o ritmo de crescimento da população idosa tem sido sistemático e constante e com isso, acredita-se que em 2025, o Brasil será a sexta maior população de idosos no mundo. Visto isso, os profissionais da área de saúde mostram o quanto à atividade física se apresenta como fator determinante para o bom processo de envelhecimento. Objetivo: realizar uma avaliação da literatura vigente sobre os efeitos do treinamento resistido em pessoas idosas. Método: para produção da compilação deste estado da arte foram realizadas buscas em bibliotecas da área de ciências biológicas, revistas eletrônicas e bases de dados virtuais. Foram encontrados 31 artigos, após analise dos títulos e resumos, 19 artigos foram excluídos. Foram selecionados 12 artigos para serem analisados para confecção do presente trabalho. Resultados: dos artigos encontrados dois $(16,67 \%)$ investigaram os efeitos do treinamento resistido sobre a mobilidade e aptidão física, três (25\%) buscaram entender o efeito do treinamento resistido de 12 semanas, três $(25 \%)$ analisaram os efeitos do treinamento resistido sobre a mobilidade, equilíbrio e outras aptidões, três artigos (25\%) investigaram o efeito do treinamento resistido com diferentes intensidades, ordem de exercícios e intervalos e somente um artigo $(8,33 \%)$ investigou sobre o funcionamento do treinamento resistido. Considerações finais: são poucos os estudos que investigam a relação treinamento resistido com patologias decorrentes desse processo. Apesar disso, os resultados encontrados são significativos e constituem um referencial para estudos posteriores desta natureza sobre a população idosa.

Palavras-chave: Treinamento resistido; Exercício; Idosos.

\section{ABSTRACT}

In Brazil the rate of the elderly population growth has been systematic and constant, and thus it is belie- ved that in 2025 Brazil will be the sixth largest elderly population in the world. Therefore health professionals show how physical activity is presented as a determining factor for the good aging process. Objetive: to conduct an assessment of the current literature on the effects of resistance training in older people. Method: searches were conducted in the biological sciences libraries, electronic journals and virtual databases. We found 31 articles, and after analysis of the titles and abstracts, 19 articles were excluded. We selected 12 articles to be analyzed in this study. Results: we found two (16.67\%) articles witch investigated the effects of resistance training on mobility and physical fitness, three (25\%) sought to understand the effect of 12 weeks resistance training, three (25\%) analyzed the effects of resistance training on mobility, balance and other skills, three (25\%) investigated the effect of resistance training with different intensities, order of exercises and intervals and only one article (8.33\%) investigated the functioning of resistance training. Closing remarks: there are few studies investigating the resistance training relationship with pathologies resulting from this process. Yet the results are significant and are a benchmark for further studies of this nature on the elderly.

Keywords: Resistance training; Exercise; Elderly.

\section{INTRODUÇÃO}

No Brasil, o ritmo de crescimento da população idosa tem sido sistemático e constante, segundo Pesquisa nacional por amostra por domicilio - PNAD 2009, realizada pelo Instituto Brasileiro de Geografia e Estatística - IBGE, o país contava com uma população de cerca de 21 milhões de pessoas com 60 anos ou mais. ${ }^{1}$ Segundo Kanso, ${ }^{2}$ ocorreu um aumento da expectativa de vida do brasileiro no período de 1940 e 2010 sendo acrescidos 30 anos na expectativa de vida da população. 
Acredita-se que, em 2025, o Brasil será a sexta maior população de idosos no mundo. ${ }^{3}$ Com o envelhecimento, várias alterações físicas e funcionais ocorrem, as quais podem potencializar o aumento das doenças crônico não transmissível (DCNT), perca de massa magra, perca do equilíbrio, dentre outros. ${ }^{4}$ Visto isso, os profissionais da área de saúde mostram o quanto à atividade física se apresenta como fator determinante para o bom processo de envelhecimento. Os benefícios da prática da atividade física estão presentes na forma dos aspectos biológicos, psicológicos e sociais, auxiliando a reintegração do idoso na sociedade, melhorando seu bem estar geral. ${ }^{5}$

A prática de exercícios físicos resulta em vários benefícios para o idoso como na melhoria de parâmetros relacionados à mobilidade funcional, ${ }^{6-8}$ hemodinâmicos ${ }^{9-11}$ e qualidade de vida, ${ }^{12-14}$ promovendo assim uma melhoria, tanto na expectativa de vida quanto em suas relações sociais.

Estudos epidemiológicos ${ }^{15,16}$ tem demonstrado a importância da prática de exercícios físicos para a manutenção das capacidades funcionais e morfológicas dos idosos, influenciando na melhoria das atividades da vida diária.

Com o envelhecimento, alguns efeitos deletérios surgem, dentre os quais a perca de massa muscular e consequentemente perda de força, funcionalidade e equilíbrio, são os mais presentes. $O$ treinamento resistido surge como uma estratégia não medicamentosa, pois, contribui para um processo de envelhecimento saudável ${ }^{7}$ e proporciona uma manutenção da autonomia. ${ }^{17-19}$ e mobilidade dos idosos, ${ }^{20}$ influenciando de maneira positiva a realização das atividades da vida diária (AVDs).

O presente estudo tem como objetivo realizar uma avaliação da literatura vigente sobre os efeitos do treinamento resistido em pessoas idosas e avaliar o estado de conhecimento transmitido por meio de artigos na literatura brasileira.

\section{METODOLOGIA}

Para produção da compilação deste estado da arte foram realizadas buscas em bibliotecas da área de ciências biológicas, revistas eletrônicas e bases de dados virtuais, como National Library of Medicine (MEDLINE), Literatura Latino-Americana e do Caribe em Ciências da Saúde (LILACS). Para isso, foram utilizados os descritores em português: "Treinamento de Resistência" e "EnveIhecimento". Durante a busca, houve o cruzamento combinado dessas palavras. Somente artigos em português publicados entre os anos de 2005 e 2015 foram considerados para elaboração do presente trabalho.

A busca dos artigos foi realizada no período de abril e maio de 2015. Foram identificados e lidos na integra por dois revisores independentes, sendo selecionados nas buscas somente artigos que apresentavam pelo menos duas palavras-chave inseridas em seu titulo e/ou resumo e que respondiam aos seguintes critérios de inclusão:

- Apresentar indivíduos com idade igual ou superior a 60 anos em sua amostra;

- Ter investigado os efeitos crônicos do exercício resistido
- Realizar intervenções por meio de treinamento resistido;

\section{RESULTADOS}

Ao realizar a busca foram encontrados 31 artigos, sendo 30 no banco de dados LILACS e um no MEDLI$\mathrm{NE}$, potencialmente relevantes. Após análise dos títulos e resumos dos trabalhos encontrados, 19 artigos foram excluídos por não responderem aos critérios de inclusão previamente estabelecidos. Portanto, foram selecionados 12 artigos para serem analisados para confecção do presente trabalho.

Dos artigos encontrados dois $(16,67 \%)$ investigaram os efeitos do treinamento resistido sobre a mobilidade e aptidão física, três (25\%) buscaram entender o efeito do treinamento resistido de 12 semanas, três $(25 \%)$ analisaram os efeitos do treinamento resistido sobre a mobilidade, equilíbrio e outras aptidões, três artigos (25\%) investigaram o efeito do treinamento resistido com diferentes intensidades, ordem de exercícios e intervalos e somente um artigo (8,33\%) investigou sobre o funcionamento do treinamento resistido (Gráfico 1).

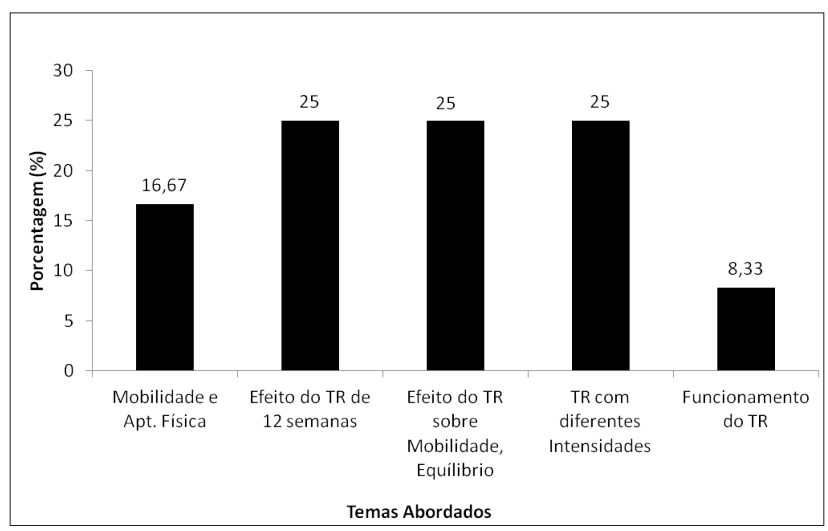

Gráfico 1 - Distribuicão dos artigos segundo os temas abordados.

Os estudos que investigaram sobre o efeito do treinamento resistido em indivíduos idosos, quatro são provenientes das regiões nordeste $(33,24)$, três da sudeste $(25 \%)$, três da região sul $(25 \%)$, um do centro-oeste $(8,33 \%)$ e um do Distrito Federal (8,33\%) (Gráfico 2). De forma geral, os estudos analisados apresentaram uma amostra com média de idade $\geq 60$ anos.

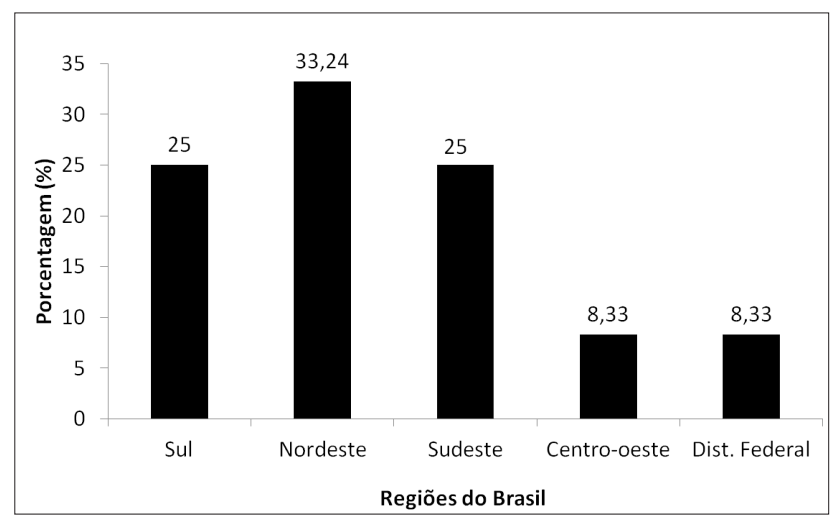

Gráfico 2 - Distribuição dos artigos de acordo com as regiões brasileiras. 
A amostra presente em dez dos 12 artigos analisados foi composta exclusivamente por mulheres, enquanto os dois restantes foram compostos, um exclusivamente por homens e o outro com amostra mista. O número de participantes dos estudos variava de dez a 45 sujeitos.

Os estudos utilizaram intensidades de treino variando de $50 \%$ a $80 \%{ }^{19,7,21}$ por se tratar de um público que requer cuidados ao se trabalhar com treinamento resistido. O número de series variava de acordo com o protocolo escolhido por cada pesquisador, variando entre oito a 15 repetições, salvo algumas exceções. ${ }^{10}$

Os métodos de treino utilizados para dois estudos foram semelhantes, ${ }^{18,19} \mathrm{com}$ a utilização de um conjunto constituído por dez exercícios, sendo realizados alternados por segmento com uma frequência de três vezes por semana (Seg. - Qua. - Sex.). Queiroz e Munaro ${ }^{21}$ fizeram uso de métodos, envolvendo duas sessões de treino por semana, pois afirmaram ao se basear em outro estudo, ${ }^{22}$ que as frequências de três e dois dias não apresentaram diferenças significativas.

\section{DISCUSSÃO}

Sabendo-se que, com o processo de envelhecimento, ocorre uma drástica diminuição de massa magra e consequentemente diminuição da força muscular, - American College of Sports Medicine ${ }^{23}$ afirma que o treinamento resistido contribui como uma estratégia não farmacológica de manutenção das capacidades físicas, podendo assim, contribuir de maneira positiva para o envelhecimento autônomo.

Tabela 1 - Aspectos metodológicos dos estudos sobre Treinamento Resistido e Idoso.

\begin{tabular}{|c|c|c|c|}
\hline Autor & População & Treinamento & Exercício \\
\hline \multirow[t]{2}{*}{ Barros et al. 2013} & Idosas & 8 semanas & 8 exercícios \\
\hline & & $3 x$ por semana & $3 \times 8$ repetições \\
\hline \multirow[t]{3}{*}{ Cunha et al. 2012} & Idosas & 8 semanas & 8 exercícios \\
\hline & & 2 semanas de adaptação & G1 - 2 x 8 repetições \\
\hline & & & G2 - 2 x 16 repetições \\
\hline \multirow[t]{2}{*}{ Cepida et al. 2013} & Idosas & 12 semanas & 6 exercícios \\
\hline & & $3 x$ por semana & $\begin{array}{c}3 \text { séries por período respiratório de } \\
5 \text { segundos }\end{array}$ \\
\hline \multirow[t]{3}{*}{ Guido et al. 2010} & Idosas & 24 semanas & 9 exercícios \\
\hline & & 3 semanas de adaptação & $3 \times 8-12$ repetições \\
\hline & & $3 x$ por semana & $60 \%$ a $80 \% 1 \mathrm{RM}$ \\
\hline \multirow[t]{2}{*}{ Jambassi et al. 2010} & Idosas & 8 semanas & 7 exercícios \\
\hline & & $3 x$ por semana & $3 \times 10-12$ repetições \\
\hline \multirow[t]{2}{*}{ Meneses-Cabral et al. 2009} & Idosas e Idosos & 16 semanas & Séries de RML \\
\hline & & Exp $2-3 x$ a $5 x$ por semana & $50 \%$ a $75 \%$ \\
\hline \multirow[t]{3}{*}{ Moura et al. 2012} & Idosas & 16 semanas & 6 exercícios \\
\hline & & $2 x$ por semana & série aumentada a cada mês \\
\hline & & & 10 repetições \\
\hline \multirow[t]{2}{*}{ Parra et al. 2009} & Idosas & 12 semanas & 9 exercícios \\
\hline & & $3 x$ por semana & $3 \times 10-15$ repetições \\
\hline \multirow[t]{2}{*}{ Pina et al. 2013} & Idosos & 7 semanas & 8 exercícios \\
\hline & & $3 x$ por semana & $3 \times 10-15$ repetições \\
\hline \multirow[t]{3}{*}{ Queiroz e Munaro, 2012} & Idosas & 8 semanas & 7 exercícios \\
\hline & & $2 x$ por semana & $2 \times 10$ repetições \\
\hline & & & $50 \%$ a $70 \%$ \\
\hline \multirow[t]{6}{*}{ Reis Filho et al, 2014} & Meia-idade e & 24 semanas & 10 exercícios \\
\hline & Idosas & 4 semanas adaptação & Adaptação \\
\hline & & $3 x$ por semana & $1^{\mathrm{a}}$ e $2^{\mathrm{a}}$ sem $\times 12$ repetições \\
\hline & & & $3^{\mathrm{a}}$ e $4^{\mathrm{a}}$ sem $\times 15$ repetições \\
\hline & & & Treino \\
\hline & & & $2 \times 10$ repetições \\
\hline \multirow[t]{3}{*}{ Terra et al. 2008} & Idosas & 12 semanas & 10 exercícios \\
\hline & & $3 x$ por semana & 3 x 12-10-8 repetições \\
\hline & & & $60 \%$ a $80 \%$ \\
\hline
\end{tabular}


Partindo deste pressuposto, o $\mathrm{ACSM}^{23}$ propôs algumas recomendações a respeito da estruturação do treinamento resistido para idosos: deve ser realizado por pelo menos duas vezes por semana com descanso de 48 horas entre as sessões, sessões com oito a dez exercícios de oito a dez repetições em cada serie, intensidade de $50 \%$ a $85 \%$ de $1 \mathrm{RM}$, cada exercício pode ser composto por uma a três séries. As sessões treino devem durar entre 30 e 60 minutos, o intervalo de repouso entre as séries deve durar de um a dois minutos.

Após análise dos artigos, observou-se que sete estudos $(58,33 \%)^{8,10,17-19,24,25}$ atenderam aos critérios de estruturação de treinamento com pesos para idosos, estabelecidos pelo ACSM, ${ }^{23}$ mostrando uma forte preocupação com a utilização de metodologias de treino já bem estabelecidas na literatura mundial, para as amostras selecionadas.

Dentre os estudos avaliados quatro $(33,33)^{7,20,21,26}$ não nortearam algumas de suas variáveis de treino segundo as recomendações do ACSM e um $(8,34 \%)^{27}$ não apresenta a descrição exata do protocolo de treino utilizado, fazendo com que seus resultados não tenham uma boa reprodutibilidade prática.

Ao analisar os artigos selecionados para análise e elaboração do presente trabalho, sete estu$\operatorname{dos}^{7,17,18,20,21,24,26}$ apresentaram em suas conclusões que o treinamento resistido melhorou a mobilidade funcional, força e potencia muscular dos idosos submetidos ao protocolo de treino de maneira significativa. Dois ${ }^{10,25}$ relataram diminuição nos níveis pressóricos dos idosos, $u^{8}{ }^{8}$ relatou que a ordem de exercícios resistidos não influenciou as respostas de composição corporal em idosos submetidos ao treinamento, um $^{27}$ relata melhorias, tanto em parâmetros cardiovasculares, quanto antropométricos, e por fim um estudo ${ }^{19}$ relatou haver melhoria na capacidade cardiorrespiratória de idosos, após treinamento resistido de 24 semanas.

Diante do grande número de estudos destinados à investigação do efeito do treinamento resistido sobre as capacidades funcionais de pessoa idosas, podemos notar uma grande preocupação em buscar maneiras de melhorar a autonomia dos idosos brasileiros. E perante os resultados obtidos em tais estudos podemos notar que os mesmos reportam uma associação positiva entre o treinamento resistido e capacidades funcionais de pessoas idosas.

\section{CONSIDERAÇÕES FINAIS}

Mesmo sabendo que o processo de envelhecimento da população brasileira é algo constante e que o treinamento resistido traz inúmeros benefícios para o organismo de idosos, ainda são poucos os estudos que investigam sua relação com patologias decorrentes desse processo.

Apesar das diferenças culturais de dimensional das amostras, das diferenças metodológicas dos estudos, os resultados encontrados são relevantes e constitui um referencial para estudos posteriores desta natureza, para melhor compreender o significado do efeito do treinamento resistido sobrea a população idosa.

\section{REFERÊNCIAS}

1. IBGE. Síntese de Indicadores Sociais: Uma Análise das
Condições de Vida da População Brasileira: 2010, p. 191.

2. Kanso S. Processo de envelhecimento populacional - um panorama mundial [Internet]. VI Workshop de Análise Ergonômica do Trabalho; III Encontro Mineiro de Estudos em Ergonomia; VIII Simpósio do Programa Tutorial em Economia Doméstica. 2012 Belo Horizonte.

3. Oliveira AC, Oliveira NM D, Arantes PMM, Alencar MA. Qualidade de vida em idosos que praticam atividade física: uma revisão sistemática. Rev Bras Geriatr Gerontol 2010;13(2):301-12.

4. Tribess S, Virtuoso JS Jr. Prescrição de Exercícios Físicos para Idosos. Rev Saúde 2005;1(2):163-72.

5. Matsudo SM, Matsudo VKR, Barros Neto TL. Atividade física e envelhecimento: aspectos epidemiológicos. Rev Bras Med Esporte 2001;7(1):1-13.

6. Silva A, Almeida GJM, Cassilhas RC, Cohen M, Peccin MS, Tufik S, Mello MT. Equilíbrio, coordenação e agilidade de idosos submetidos à prática de exercícios físicos resistidos. Rev Bras Med Esporte 2008;14(2):88-93.

7. Moura M, Pedrosa M, Costa E, Bastos Filho P, Sayão L, Sousa T. Efeitos de exercícios resistidos, de equilíbrio e alongamentos sobre a mobilidade funcional de idosas com baixa massa óssea. Rev Bras Ativ Fis Saúde 2013;17(6):474-84.

8. Pina FLC, Nascimento MA, Januário RSB, Gerage AM, Oliveira AR, Cyrino ES. Influência da ordem de exercícios com pesos sobre a composição corporal em homens idosos. Rev Educ Fís/UEM 2013;24(3):443-51.

9. Costa JBY, Gerage AM, Gonçalves CGS, Pina FLC, Polito MD. Influência do estado de treinamento sobre o comportamento da pressão arterial após uma sessão de exercícios com pesos em idosas hipertensas. Rev Bras Med Esporte 2010;16(2):103-6.

10. Cunha, Eline Silva. Efeito do treinamento resistido na pressão arterial e capacidade funcional de idosas hipertensas. [Dissertação de Mestrado]. Natal: Universidade Federal do Rio Grande do Norte; 2010.

11. Krinski K, Elsangedy HM, Soares IA, Buzzachera CF, Campos W, Silva SG. Efeitos cardiovasculares agudos do exercício resistido em idosas hipertensas 2008;30(2):107-12. doi: 10.4025/actascihealthsci.v30i2.428

12. Aguiar B, Moraes $H$, Silveira $H$, Oliveira $N$, Deslandes $A$, Laks J. Efeito do treinamento físico na qualidade de vida em idosos com depressão maior. Rev Bras Ativ Fís Saúde 2014;19(2):205-14. doi: 10.12820/RBAFS.V.19N2P205

13. Silva MF, Goulart NBA, Lanferdini FJ, Marcon M, Dias CP. Relação entre os níveis de atividade física e qualidade de vida de idosos sedentários e fisicamente ativos. Rev Bras Geriatr Gerontol 2012;15(4):634-42. doi: 10.1590/S180998232012000400004

14. Cheik NC, Reis IT, Heredia RAG, Ventura ML, Tufik S, Antunes HKM, Mello MT. Efeitos do exercício físico e da atividade física na depressão e ansiedade em indivíduos idosos. Rev Bras Ci e Mov 2003;11(3):45-52. doi: 10.18511/rbcm.v11i3.509

15. Lima-Costa MF, Barreto SM, Giatti L. Condições de saúde, capacidade funcional, uso de serviços de saúde e gastos com medicamentos da população idosa brasileira: um estudo descritivo baseado na Pesquisa Nacional por Amostra de Domicílios. Cadernos de Saúde Pública 2003;19(3):735-43. doi: 10.1590/S0102-311X2003000300006

16. Ramos LR, Rosa TEC, OLiveira ZM, Medina MCG, Santos FRG. Perfil do idoso em área metropolitana na região sudeste do Brasil: resultados de inquérito domiciliar. Rev Saúde Pública 1993;27(2):87-94. doi: 10.1590/S0034- 


\section{3}

17. Parra MT, Parra SA, Oliveira AR, Greguol M. Influência do treinamento com pesos sobre a capacidade funcional de mulheres idosas. RBCM 2009;17(3):32-8. doi: 10.18511/ rbcm.v17i3.956

18. Reis Filho AD, Vieira Junior RC, Voltarelli FA. Treinamento específico de flexão e extensão do punho não aumentou a força de preensão manual de idosas. Rev Bras Ciência e Movimento 2014;22(2):87-95. doi: 10.18511/rbcm.v22i2.4397

19. Guido M, Lima RM, Benford R, Leite TKM, Pereira RW, Oliveira RJ. Effects of 24 weeks of resistance training on aerobic fitness indexes of older women. Rev Bras Med Esporte 2010;16(4):259-63. doi: 10.1590/\$151786922010000400005

20. Cepeda CC, Rodacki ALF, Persch LN, Silva PP, Buba S, Dressler VF. Efeitos do método isostretching sobre parâmetros morfológicos e sobre um conjunto de testes motores em idosas. Rev Bras Cineantropom Desempenho Hum 2013;15(5):604-15. doi: 10.5007/1980-0037.2013v15n5p604

21. Queiroz CO, Munaro HLR. Efeitos do treinamento resistido sobre a força muscular e a autopercepção de saúde em idosas. Rev Bras Geriatr Gerontol 2012;15(3):547-53. doi: 10.1590/S1809-98232012000300015

22. Stadler LV, Stubbs NB, Vukovich MDA. Comparison of a 2-day and 3-day per week resistance training program on strength gains in older adults. Med Sci Sports Exerc 1997;29(5):254.

23. American College of Sports Medicine, Chodzko-Zajko
WJ, Proctor DN, Fiatarone Singh MA, Minson CT, Nigg CR, Salem GJ, Skinner JS. American College of Sports Medicine position stand. Exercise and physical activity for older adults. Med Sci Sports Exercise 2009;41(7):1510-30. doi: 10.1249/MSS.0b013e3181a0c95c.

24. Barros CC, Caldas CP, Batista LA. Influência do treinamento da potência muscular sobre a capacidade de execução de tarefas motoras em mulheres idosas; Influence of muscle power training on the ability to implement motor tasks for older women. Rev Bras Geriatr Gerontol 2013;16(3):603-13. doi: 10.1590/S1809-98232013000300017

25. Terra DF, Mota MR, Rabelo HT, Bezerra LMA, Lima RM, Ribeiro AG, VinhalPH, DiasRMR, SilvaFM. Redução da pressão arterial e do duplo produto de repouso após treinamento resistido em idosas hipertensas. Arq Bras Cardiol 2008;91(5):274-9. doi: 10.1590/S0066-782X2008001700003

26. Jambassi Filho JC, Gurjão ALD, Gonçalves R, Barboza BHV, Gobbi S. O Efeito de diferentes intervalos de recuperação entre as séries de treinamento com pesos, na força muscular em mulheres idosas treinadas. Rev Bras Med Esporte 2010;16(2):112-5. doi: 10.1590/S151786922010000200007

27. Menezes-Cabral RL, Silva-Dantas PM, Montenegro-Neto AM, Knackfuss MI. Efeitos de diferentes treinamentos e estilos de vida nos indicadores antropométricos e cardiocirculatórios no envelhecimento. Rev Salud Pública 2009;11(3):359-69. doi: 10.1590/S0124-00642009000300005 